\title{
Identification of Sarcopenia Risk in Oncology Outpatients using the SARC-F Method
}

\author{
Ana Paula Noronha Barrére ${ }^{1}$, Giovanna Guimarães Lopes ${ }^{1}$, Thaisa de Assis ${ }^{1}$, \\ Silvia Maria Fraga Piovacari ${ }^{1}$, Diogo Oliveira Toledo ${ }^{2 *}$ \\ ${ }^{1}$ Nutrition Department of Hospital Israelita Albert Einstein, São Paulo, Brazil \\ ${ }^{2}$ Nutrologist physician at Hospital Israelita Albert Einstein, São Paulo, Brazil
}

\begin{abstract}
Received: June 08, 2018; Accepted: August 03, 2018; Published: August 7, 2018
*Corresponding author: Diogo 0 Toledo, Avenida Albert Einstein, 627/701, Morumbi, São Paulo - Brazil, ZC: 05651901, E-mail: diogootoledo@ gmail.com
\end{abstract}

\begin{abstract}
Introduction: Sarcopenia is a common syndrome of highly catabolic diseases like cancer. Maintaining adequate body composition is fundamental for oncology patients' best prognosis. Existing tools to help in early identification of sarcopenia are limited and impractical in outpatient care. The SARC-F questionnaire, validated by Malmstrom et al, in order to screen the risk of sarcopenia can help on the early diagnosis and intervention. The objective of this study was to evaluate the sarcopenia risk in oncology outpatients by SARC-F tool, and to analyze its relationship with the Body Mass Index (BMI) and treatment toxicity.

Method: A prospective study was carried out to 52 elderly oncology outpatients of a tertiary hospital clinic in São Paulo, Brazil. The patients' risk of sarcopenia was assessed by SARC-F questionnaire, through direct interview, at the time of radiotherapy or chemotherapy. The demographic data collected were sex, age, diagnosis, weight, presence of toxicity and BMI, (classified according to SABE PAHO, 2003). Toxicity classification was performed according to the Common Toxicity Criteria of the National Cancer Institute.

Results: In the study, most patients were males ( $\mathrm{n}=32,61.53 \%)$, mean age was 72 years-old ( \pm 8.4 ), weight showed mean of $72.9 \mathrm{~kg}( \pm 12.9)$ and BMI of $25,65 \mathrm{~kg} / \mathrm{m}^{2}( \pm 3,85)$, classifying as eutrophic $55.7 \%(\mathrm{n}=29), 19.2 \%$ underweight $(\mathrm{n}=10)$ and $25 \%$ overweight $(\mathrm{n}=13)$. Of the patients, 34 were undergoing to chemotherapy $(65.38 \%)$ and 18 to radiotherapy $(34.19 \%)$. The most frequent neoplasms were: prostate $(\mathrm{n}=8,15,38 \%)$, breast $(n=7,13,46 \%)$, lung $(n=7,13,46 \% 62 \%)$ and others $(n=25 ; 48.08 \%)$. Toxicity was presented in $55.76 \%$ of patients ( $n=29)$, being the most common inappetence $(n=9 ; 31.03 \%)$; nausea $(n=5 ; 17.24 \%)$ and diarrhea $(n=5 ; 17.24 \%)$. Among the patients who presented a risk for sarcopenia $(\mathrm{n}=6 ; 11.53 \%)$, according to SARC-F, the majority were female $(\mathrm{n}=4 ; 66.6 \%)$ aged 80 to 90 years-old $(\mathrm{n}=3 ; 50.0 \%)$. Of patients at sarcopenia risk, $33.3 \%$ were underweight, $50.0 \%$ were eutrophic and $16.6 \%$ were overweight according to the BMI classification. Regarding toxicity, $66.6 \%(\mathrm{n}=4)$ of the sarcopenia risk patients presented toxicity, $50.0 \%$ with inappetence $(n=2)$ and $50.0 \%$ with nausea or diarrhea $(n=2)$.

Conclusion: There was a risk of sarcopenia in $11.53 \%$ of the patients $(n=6)$, but it was not related to BMI. As for toxicity, the tool revealed a positive relation regarding sensitivity but without statistical relevance. SARC-F is a quick and simple screening method for sarcopenia, which can be applied by any healthcare professional. However, further studies are needed for application in clinical oncology area.
\end{abstract}

Keywords: Sarcopenia; Oncology; Outpatients

\section{Introduction}

Sarcopenia is a syndrome characterized by a gradual and generalized process of lean mass loss and strength reduction, associated to functionality decrease [1]. The syndrome has primary origin when triggered by mechanisms primarily correlated with age, involving hormones, apoptosis and mitochondrial dysfunctions. While its secondary origin is the result of organic failure, inflammatory, malignant or endocrine diseases. A prevalence of $50 \%$ sarcopenia is estimated in the elderly over 80 years-old [1, 2].

Cancer is characterized by uncontrolled cell growth. Metabolic changes such as exacerbated energy expenditure, inflammation, anorexia and the tumor mass metabolism contribute to the quick nutritional status deterioration due to inflammatory mediators and adverse effects of treatment $[1,3,5,6]$.

The metabolic disturbances caused by the tumor mass that contribute to patient's malnutrition and sarcopenia include:

- Increased production of inflammatory cytokines, such as TNF- $\alpha$ and IL-6. In excess, these cytokines trigger the release of myofibrillar proteins that inhibit AKT / mTOR pathway signaling, thus contributing to muscle atrophy. In parallel to this, occurs MuRF-1 (muscle RING finger-containing protein 1) and MAFbx (atrogin) protein activation, both of which potentiate muscle loss and functionality reduction. Inflammatory cytokines further reduce appetite, contributing to food inappetence $[5,6]$.

- Lipolysis induction by the adrenergic metabolic pathway 
that activates and stimulates protein kinase A, Adipose Tissue Triglyceride Lipase (ATGL) and Hormone-Sensitive Lipase (HSL), which release into the plasma free-chain fatty acid to be used as energy supply [6].

For these reasons, attention to patient' nutritional status is essential for treatment good prognosis and quality of life recovery. Uncontrolled levels of protein depletion may impair or even discontinue anticancer treatments, since chemotherapeutics agents are metabolized in muscle tissue and its dosage is calculated according to muscle mass at the treatment beginning $[5,6]$. Approximately $20 \%$ of cancer patient deaths are secondary to malnutrition. Patients with muscle mass depletion present a greater risk of treatment toxicity, lower survival and worse clinical outcome $[7,8]$.

Treatment toxicity is measured by gastrointestinal symptoms, and classification is performed based on the extent to which they manifest themselves. According to the Brazilian Institute of Oncology Nutrition (IBNO), the most common toxicities are anorexia (26\%), nausea and vomiting (33\%), xerostomia (19\%), odynophagia (11\%) and diarrhea (16\%) [5, 9].

Early identification of patients at risk of malnutrition and sarcopenia is essential to enable appropriate intervention and improve nutritional status. Therefore, the aim of the present study was to evaluate the sarcopenia risk in oncological outpatient population using the SARC-F questionnaire; and to analyze if there is an association between SARC-F and Body Mass Index (BMI) classification and with the SARC-F and toxicity symptoms during the anticancer treatment.

\section{Methods}

A prospective, descriptive study carried out in a private hospital of São Paulo, Brazil, from April to May 2017. Were included elderly oncology outpatients ( $\geq 60$ years-old). Exclusion criteria were physical and/or mental incapacity to perform the requested questionnaire.

The questionnaire used in this study was the SARC-F in the validated version for Portuguese language, indicated for screening the risk of sarcopenia. It is composed of five objective questions, self-reported by the patient in direct interview mode that was applied during oncology treatment (chemotherapy and radiotherapy). SARC-F questionnaire includes five components: strength, assistance walking, rise from a chair, climb stairs, and falls. The scale scoring range from 0 to 10 (i.e. $0-2$ points for each component; $0=$ best performance to $10=$ worst performance) and classify the patient as symptomatic ( $\geq 4$ points) or healthy $(0$ to 3 points) (Chart 1) [10].

The data collected were sex, age, weight, height, body mass index (BMI), diagnosis, type of treatment, presence and symptom of toxicity.

\begin{tabular}{|lll|}
\hline Component & Question & Scoring \\
\hline Strength & How much difficulty do you have & None $=0$ \\
& in lifting and carrying 10 & Some $=1$ \\
& pounds? & A lot or unable $=2$ \\
\hline Assistance in & How much difficulty do you have & None $=0$ \\
walking & walking across a room? & Some $=1$ \\
& & A lot, use aids, or unable $=2$ \\
\hline Rise from a chair & How much difficulty do you have & None $=0$ \\
& transferring from a chair or bed? & Some $=1$ \\
& & A lot or unable without help =2 \\
\hline Climb stairs & How much difficulty do you have & None $=0$ \\
& climbing a flight of 10 stairs? & Some $=1$ \\
& & A lot or unable $=2$ \\
\hline Falls & How many times have you fallen in & None $=0$ \\
& the past year? & $1-3$ falls $=1$ \\
& & 4 or more falls $=2$ \\
\hline
\end{tabular}

Chart 1: SARC-F screening for sarcopenia. Adapted from Malmstrom et al (2013)

The BMI results for elderly patients ( $\geq 60$ years-old) were classified by using the SABE/OPAS (Survey on Health, Welfare and Aging by Pan American Health Organization) classification of 2003. Toxicity classification was performed according to the Common Toxicity Criteria of the National Cancer Institute (version 2.0) [5, 11, 12, 13].
Mean, median and standard deviation were calculated for the quantitative variables and absolute frequencies or percentages for the qualitative variables as appropriate. Pearson' qui-square test was applied for the categorical variables, with p-value lower or equal to 0.05 considered as statistically significant. Simple 
linear regression was used to establish possible associations between variables and SARC-F. Data were analyzed by using Microsoft Office Excel 2010 version.

The uniterms utilized were sarcopenia, oncology, oncology outpatient.

\section{Results}

The random and non-probabilistic sample consisted of 52 elderly individuals, with 32 male (61.5\%), mean age of 72 years $( \pm$ $8.4)$, mean weight of $72,9 \mathrm{~kg}( \pm 12.9)$ and mean BMI of $25.65 \mathrm{~kg} /$ $\mathrm{m}^{2}( \pm 3.85)$. According to BMI, there was 10 underweight $(19.2 \%)$, 29 eutrophic (55.8\%) and 13 overweight (25\%) patients.

Toxicities were related by $55.8 \%$ of patients. The adverse events most frequently reported by patients were appetite loss (31.03\%), nausea (17.24\%), diarrhea (24\%).

In the study, 34patients were undergoing to chemotherapy $(65.4 \%)$ and 18 to radiotherapy (34.6\%). The type of neoplasia presented a prevalence of $15.4 \%$ to prostate $(n=8), 13.5 \%$ to breast $(n=7), 13.5 \%$ to lung $(n=7), 9.6 \%$ to rectum $(n=5)$ and $48.1 \%$ to other types $(n=25 ; 48,1 \%)$ Table 1.

\begin{tabular}{|c|c|c|}
\hline Variables & $n=52$ & $\%$ \\
\hline \multicolumn{3}{|l|}{ Sex } \\
\hline Female & 20 & 38,5 \\
\hline Male & 32 & 61,5 \\
\hline \multicolumn{3}{|l|}{ Age (years) } \\
\hline 60 to 69 & 23 & 44,2 \\
\hline 70 to 79 & 17 & 32,7 \\
\hline 80 to 89 & 11 & 21,2 \\
\hline 90 to 100 & 1 & 1,9 \\
\hline \multicolumn{3}{|l|}{ Type of tumor } \\
\hline Adenoneuroendocrine & 1 & 1,9 \\
\hline Esophagus & 1 & 1,9 \\
\hline Gastric & 1 & 1,9 \\
\hline Glioblastoma & 3 & 5,8 \\
\hline Liver & 1 & 1,9 \\
\hline Lymphoma & 1 & 1,9 \\
\hline Follicularlymphoma & 2 & 3,8 \\
\hline Hodgkin's lymphoma & 1 & 1,9 \\
\hline Non Hodgkin's lymphoma & 2 & 3,8 \\
\hline Tongue & 1 & 1,9 \\
\hline Breast & 7 & 13,6 \\
\hline Myelodysplasia & 2 & 3,8 \\
\hline Myeloma & 2 & 3,8 \\
\hline Ovarian tumor & 1 & 1,9 \\
\hline
\end{tabular}

\begin{tabular}{|c|c|c|}
\hline Pancreas & 2 & 3,8 \\
\hline Peritoneum & 1 & 1,9 \\
\hline Prostate & 8 & 15,5 \\
\hline Lung & 7 & 13,6 \\
\hline Rectum & 5 & 9,7 \\
\hline Soft tissue sarcoma & 2 & 3,8 \\
\hline Brain & 1 & 1,9 \\
\hline \multicolumn{3}{|l|}{ Treatment type } \\
\hline Chemotherapy & 34 & 65,4 \\
\hline Radiotherapy & 18 & 34,6 \\
\hline \multicolumn{3}{|l|}{ SARC-F } \\
\hline Sarcopenia risk (6-10 points) & 6 & 11,5 \\
\hline $\begin{array}{l}\text { No sarcopenia risk (0-5 } \\
\text { points) }\end{array}$ & 46 & 88,5 \\
\hline \multicolumn{3}{|l|}{ Body Mass Index (BMI) } \\
\hline$\leq 23$-Underweight & 10 & 19,2 \\
\hline 23,0 to 28,99 - Eutrophia & 29 & 55,8 \\
\hline$\geq 29.0$ - Overweight & 13 & 25,0 \\
\hline \multicolumn{3}{|l|}{ Toxicity } \\
\hline No & 24 & 46,2 \\
\hline Yes & 28 & 53,8 \\
\hline \multicolumn{3}{|l|}{ Adverse symptoms } \\
\hline Inappetence & 9 & 17,3 \\
\hline Diarrhea & 5 & 9,6 \\
\hline Nausea & 5 & 9,6 \\
\hline Constipation & 4 & 7,7 \\
\hline Abdominal colic & 1 & 1,9 \\
\hline Dysphagia & 1 & 1,9 \\
\hline Tastedisorders & 1 & 1,9 \\
\hline Vomiting & 2 & 3,8 \\
\hline
\end{tabular}

According to SARC-F, 6 patients $(11,5 \%)$ were at sarcopenia risk, of these $66,6 \%$ were female and most aged 80 to 90 years $(\mathrm{n}=3 ; 50,0 \%)$ Table 2. The BMI classification was compared to sarcopenia ( $\mathrm{n}=6): 2(33.3 \%)$ underweight, $3(50.0 \%)$ eutrophic, $1(16.6 \%)$ overweight Table 3 (Graphic 1).

According to BMI, $57.14 \%$ of the patients were eutrophic. Patients presenting toxicity were $53.8 \%$ and the most common of them was inappetence (43.8\%). Of the 28 patients who presented toxicity, only 7 (25\%) were classified at nutritional risk by BMI (underweight), of these the most common symptoms were constipation and inappetence (28.57\%) Table 4 (Graphic 2). 
Table 2: SARC-F's performance for sarcopenia screening

\begin{tabular}{|c|c|c|c|c|c|c|c|c|}
\hline \multirow[b]{3}{*}{ Age } & \multicolumn{4}{|c|}{ Risk for sarcopenia $(n=6)$} & \multicolumn{4}{|c|}{ No risk for sarcopenia $(n=46)$} \\
\hline & \multicolumn{2}{|c|}{$\begin{array}{c}\text { Female } \\
(n=4)\end{array}$} & \multicolumn{2}{|c|}{$\begin{array}{c}\text { Male } \\
(n=2)\end{array}$} & \multicolumn{2}{|c|}{$\begin{array}{l}\text { Female } \\
(n=16)\end{array}$} & \multicolumn{2}{|c|}{$\begin{array}{c}\text { Male } \\
(n=30)\end{array}$} \\
\hline & $\mathbf{n}$ & (\%) & $\mathbf{n}$ & (\%) & $\mathbf{n}$ & (\%) & $\mathbf{n}$ & (\%) \\
\hline 60 to 69 & 1 & 25,0 & 0 & - & 9 & 56,3 & 13 & 43,3 \\
\hline 70 to 79 & 2 & 50,0 & 0 & - & 6 & 37,5 & 9 & 30,0 \\
\hline 80 to 89 & 1 & 25,0 & 2 & 100,0 & 1 & 6,3 & 7 & 23,3 \\
\hline 90 to 100 & 0 & - & 0 & - & 0 & - & 1 & 3,3 \\
\hline TOTAL & 4 & 100,0 & 2 & 100,0 & 16 & 100,0 & 30 & 100,0 \\
\hline
\end{tabular}

Table 3: Description of BMI and SARC-F classification $(n=52)$

\begin{tabular}{|c|c|c|}
\hline & Sarcopenia risk & No sarcopenia risk \\
\hline Underweight & $2(4 \%)$ & $8(15 \%)$ \\
\hline Eutrophia & $3(6 \%)$ & $26(50 \%)$ \\
\hline Overweight & $1(2 \%)$ & $12(23 \%)$ \\
\hline
\end{tabular}

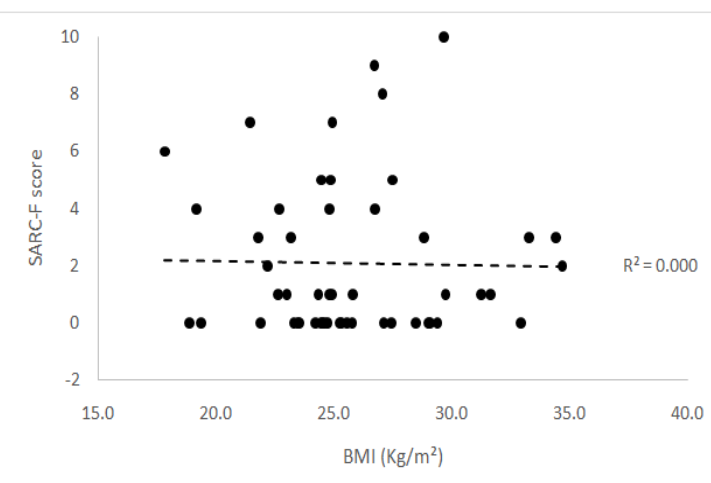

Graphic 1: Scatterplot of SARC-f versus BMI

Table 4: Correlation between the presence of toxicity and SARC-F classification $(\mathrm{n}=52)$

\begin{tabular}{|c|c|c|c|}
\hline & $\begin{array}{c}\text { Sarcopenia } \\
\text { risk }\end{array}$ & $\begin{array}{c}\text { No sarcopenia } \\
\text { risk }\end{array}$ & p-value \\
\hline Toxicity presence & 2 & 22 & 0,50 \\
\hline No toxicity & 4 & 24 & \\
\hline
\end{tabular}

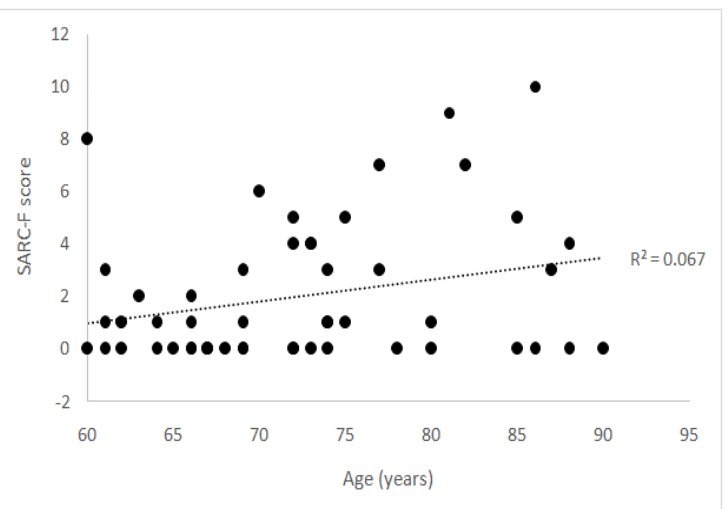

Graphic 2: Scatterplot of SARC-f versus Age

\section{Discussion}

Barbosa et, al. (2016) reported low sensitivity but high specificity to identify sarcopenia risk by the SARC-F questionnaire in its isolate application. The factors that contribute to this fact are related to the questions that mainly evaluate the performance, creating a gap regarding the evaluation of the lean mass loss, given that these are the two main strands for the sarcopenia characterization, according to The European Group of Sarcopenia in Elderly (EWGSOP) [1, 4]. The group proposed a possibility to make SARC-F more sensitive, by using calf circumference (cut-off point: $33 \mathrm{~cm}$ for women and $34 \mathrm{~cm}$ for men), which is considered gold standard for the elderly. However, in oncology patients anthropometric measurements may be influenced by non-nutritional factors, such as edema due to, for example, drugs administered during treatment $[4,8]$.

The EWGSOP offers other options for muscle mass measure like computed tomography, considered a gold standard method, electric bioimpedance and others [1]. Some of these technologies to analyze body composition are not financially feasible to identify sarcopenia $[5,11]$. 
Dynamometry is an effective measure for all age groups and provides support for both, isolated muscle mass and muscle performance, being an extremely valuable aid to the SARC-F questionnaire. In addition, completing the questionnaire with these instruments helps to reduce the subjectivity of selfreporting [4].

Regarding the results obtained in the study $(11.5 \%$ of the sample with risk of sarcopenia), it is worth to mentioning that the tumors with the highest incidence were: prostate, breast and lung. It is known that neoplasias with the highest nutritional risk are those located in gastrointestinal tract, which is a possible justification for the low prevalence of the risk of sarcopenia [9].

Concerning to toxicity, the study showed SARC-F pre disposition for specificity of toxicity. It suggests that SARC-F may be assertive about the risk of sarcopenia, since sarcopenia patients have a greater chance of presenting adverse effects to treatment.

In addition, it is important to emphasize that BMI is a pattern of population analysis, widely used in the clinical practice. However, it may not to be a sensitive parameter, especially in the elderly oncologic population where the height used is usually the one reported and the weight is altered due to anticancer treatments $[1,2]$.

The study presented biases, as the small sample with great heterogeneity, the different types of neoplasias presented and the non-control of the number of treatment sessions that the patients underwent at the time of collection. Justifying the intention of the study, to portray the heterogeneity in daily care reality of an oncological clinic.

\section{Conclusion}

The risk of sarcopenia was present in $11.53 \%$ of the sample $(\mathrm{n}=6)$, but it did not demonstrated relation with BMI or toxicity. SARC-F is a quick and simple screening method for sarcopenia, which can be applied by any healthcare professional. However, further studies are needed for application in clinical oncology area. Studies with SARC-F associated with tools to measure muscle mass and strength, such as Handgrip or thumb adductor muscle thickness (EMAP), are suggested.

\section{References}

1. Cruz-Jentoft AJ, Baeyens JP, Bauer JM, Boirie Y, Cederholm T, Landi F, et al. Sarcopenia: European consensus on definition and diagnosis. Age Ageing. 2010;39(4):412-423. doi: 10.1093/ageing/ afq034

2. Mitchell WK, Williams J, Atherton P, Larvin M, Lund J, Narici M. Sarcopenia, dynapenia, and the impact of advancing age on human skeletal muscle size and strength; a quantitative review. Front Physiol. 2012;3:260. doi: 10.3389/fphys.2012.00260
3. Graf CE, Herrmann FR, Spoerri A, Makhlouf AM, Sørensen TIA, Ho $\mathrm{S}$, et al. Impact of body composition changes on risk of all-cause mortality in older adults. Clin Nutr. 2016;35(6):1499-1505. doi: 10.1016/j.clnu.2016.04.003

4. Barbosa-Silva TG, Menezes AM, Bielemann RM, Malmstrom TK, Gonzalez MC. Enhancing SARC-F: Improving Sarcopenia Screening in the Clinical Practice. Journal of the American Medical Directors Association. 2016;17(12):1136-1141.

5. Rezende ACP, Barrére APN, Todaro J, Tanaka M. In: Nutritional Guide in Oncology: Barrere APN, Pereira A, Hamerschlak N, Piovacari SMF. 2017:1(1):128-139.

6. Vega Maria Cecília Monteiro Dela, Laviano Alessandro, Pimentel Gustavo Duarte. Sarcopenia and toxicity mediated by chemotherapy. Einstein (São Paulo). 2016;14(4):580-584. doi: 10.1590/S167945082016MD3740

7. Coronha, Ana Lúcia; Camilo, Maria Ermelinda; Ravasco, Paula. The importance of body composition in the oncological patient: What is the Evidence? Acqua Médica Portuguesa, Lisboa. 2011;769-788.

8. Pinho NB, Oliveira GPC, Correia MITD, Oliveira AGL, Souza CM, Cukier C, et al. Nutritional Therapy in Oncology - Brazilian Society of Parenteral and Enteral Nutrition Brazilian Association of Nutrology, 2011.

9. Instituto Nacional de Câncer José Alencar Gomes da Silva. Nutrition guide for patients and caregivers: guidelines for patients / Instituto Nacional de Câncer José Alencar Gomes da Silva. 2015;3(1):7-8.

10. Malmstrom TK, Morley JE. SARC-F: A Simple Questionnaire to Rapidly Diagnose Sarcopenia. J Am Med Dir Assoc. 2013;14(8):531532. doi: 10.1016/j.jamda.2013.05.018

11. Barrere, Ana Paula, Horie, Lilian, Nogueira, Priscila, et al. In: Multiprofessional Nutritional Therapy Team: EMTN in practice: Piovacari, Silva; Toledo, Diogo; Figueredo, Evandro. Atheneu, 2017;1(1):14-56.

12. Rossi, Luciana, Caruso, Lucia, Galante, Andrea Polo. Nutritional assessment: New perspectives. São Paulo: Roca, 2010.

13.Gaikwad NR, Gupta SJ, Samarth AR, Sankalecha TH. Handgrip dynamometry: a surrogate marker of malnutrition to predict the prognosis in alcoholic liver disease. Ann Gastroenterol. 2016;29(4):509-514. doi: 10.20524/aog.2016.0049 